\title{
Numerical modelling of T-pipe joints forming
}

\author{
JarosławBartnicki ${ }^{1,}$ \\ ${ }^{1}$ Lublin University of Technology, 36 Nadbystrzycka, 20-618 Lublin, Poland
}

\begin{abstract}
This paper presents results of numerical calculations of T-pipe joints (tee) forming process for a wide scope of industrial applications. FEM analyses were conducted for the chosen process technological parameters and tools designs. The kinematics of metal flow in the area of the formed flanges was analysed. Distributions of stresses, strains and damage criterion during forming were determined. Calculated values of forces and moments acting on tools and workpieces allow for designing of tools geometry for experimental verification of the proposed forming process.
\end{abstract}

Keywords: FEM, design optimization, cold forming

\section{Introduction}

The solutions for different liquid, sand, gases transportations are very different. Many of them concern applications of different advanced polymer technologies but for high pressure pipe ducts steel materials are commonly applied. In this case it is extremely important to preserve good sealing of designed pipelines. Different welding technologies are normally used for connections making but especially difficult case among them is Tpipe joints (tee) forming and welding with another pipe structures.

For a wide scope of application connections made with T-pipe joints are realized by drilling the wholes inside one pipe and its welding into another. For increasing mechanical characteristics and life time of these connections flanging technology is applied. This solution is favourable for pressure distribution and makes the welding processes easier for making and verifying - Fig. 1. The proposed solution is also less material consuming comparing with traditional one and it can be also applied in existing installations using portable devices for drilling and tee making. Typical applications areas are in food processing, pharmaceutical, pulp and paper industry, stainless steel piping systems, water treatment, shipbuilding and conventional and nuclear submarines[1].

The proposed solution for bigger pipe installations must be equipped with special machine for tee making. This aggregate consist of drilling section for elliptic hole making, and special flanging head. Combining rotations and axial movement of this element results in flanges (collars) creation. For smaller pipes tee making can be realized by means of portable tools because this process does not require application of bigger values of forces and torques.

The observations of this problematic and numerical verification of material flow and technological parameters are fundamental for planned new devices for
T-pipe joints development. The most important for new solution designing is tools geometry and load parameters knowledge combined with final parts dimensions accuracy [2-4].

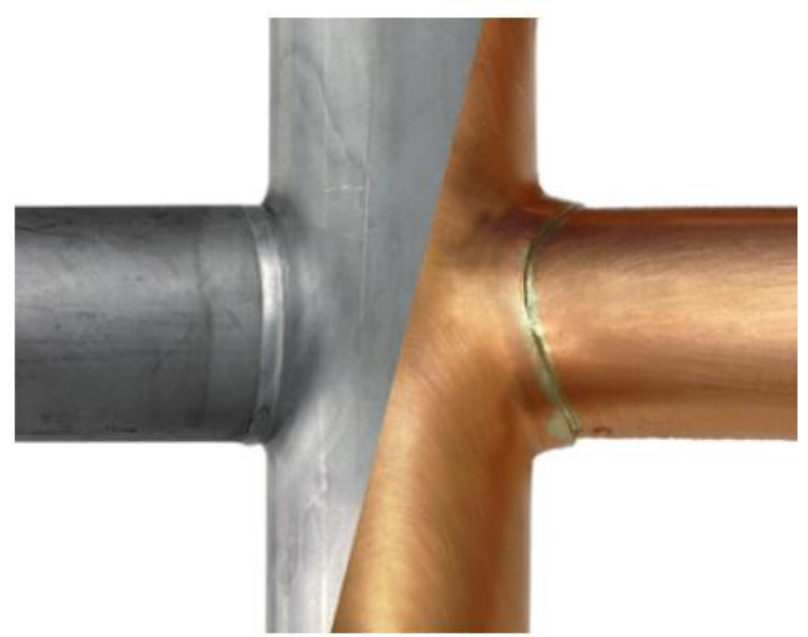

Fig. 1.The example of traditional (left) and formed by flanging T-pipe joint (right) [1]

\section{Numerical modelling}

Numerical calculations of proposed scope of flanging processes were realized using Deform 3D software. In numerical models were applied pipes with external diameter $\varnothing 150$ and $\varnothing 300 \mathrm{~mm}$ and created collars with diameters $\varnothing 50$ and $\varnothing 100 \mathrm{~mm}$.

\subsection{Numerical models}

In numerical simulations the pipes with the mentioned above two different external diameters and two collars were considered. As the material model weldable fine-grain structural steel for pressure vessels $\mathrm{P} 355 \mathrm{NH}$ was used [2]. For calculations in the cold metal

Corresponding author: j.bartnicki@pollub.pl 
forming conditions all tools and workpieces have the same initial temperature $\mathrm{T}=20^{\circ} \mathrm{C}$. The constant friction model was assumed for all cases with friction factor $\mathrm{m}=$ 0.25 , as the processes are realized with good lubrication.

Some calculations were realized with workpiece mounted inside the jaws with diameter corresponding to the pipe dimensions. After verification of state of stress and strain, the workpiece were limited to the halfpipes fixed by boundary conditions at the external edges. The workpiece was meshed by tetra - type elements with refinement of forming zone by three times smaller ones with minimum 5 elements on wall thickness as assumption. This solution permits to realize simulation faster.

For the proper circular collar shape forming the initial holes inside the pipes was designed as elliptic calculating geometrically created collar. For example, the collar $\varnothing 50 \mathrm{~mm}$ formed into the pipe $\varnothing 150 \mathrm{~mm}$ required initial elliptic whole $30 \times 37 \mathrm{~mm}$. The examples of worked out models of the analysed cases are presented in Fig. 2. The first model consists of formed pipe with initial elliptic whole, outer pipe as mounting for workpiece and two bars - tools which flange the collar. In the second, developed model physical fixation is replaced by boundary conditions with fixed edges (red nodes in Fig 2).
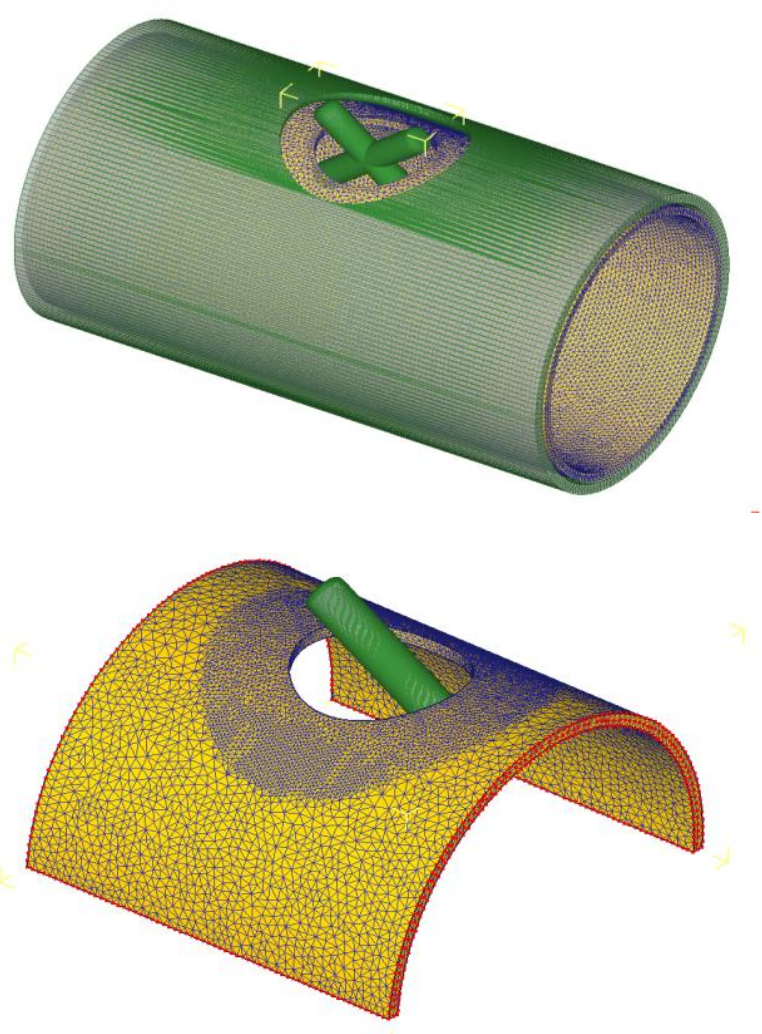

Fig.2. Worked out numerical models for calculations with physical pipe stabilization (above) and with boundary conditions with fixed displacement (below)

For time calculation optimization in later realized simulation the box with fine mesh was applied. These modifications help to make simulation faster. All these changes were made after state of stress verifications with the first model without any changes.

\subsection{Feed rates and tools configurations}

The technological values crucial for the analysed cases were feed rates $\mathrm{f}$ written in $\mathrm{mm}$ of working tools movement per rotations. This parameter is strictly combined with dimensional accuracy and effective time of realized collar tee connections.

Analysing accessible solution [1] it was assumed to apply solution with $1,2,3$ and 4 rotating and axially moving tools. The forming tools - bars have the same diameter $\varnothing 10 \mathrm{~mm}$ and specially rounded ends. According to the chosen option, the feed rates applied in calculations were $0.5 ; 1.0$ and $2 \mathrm{~mm}$ per one rotation.

Another very important technological parameter in this forming process was tools - bars inclination angle $\alpha$. The values for this parameter were taken within the range of $\alpha=(35 \div 60)^{\circ}$, which results in collar flanging mechanism and final joint edge shape. This parameter also changes final strain distribution. Smaller inclination angle and feed rates lead to obtaining bigger values of strain with significant part of redundant strains.

\section{Simulations results}

\subsection{States of effective stress and strain}

During numerical simulations realized with the presented assumptions and technological parameters it was possible to verify distributions of stress and strain during collars flanging. In Fig. 3 and Fig. 4 the results of calculations for $\varnothing 50$ collar forming into $\varnothing 150 \mathrm{~mm}$ pipe are presented.

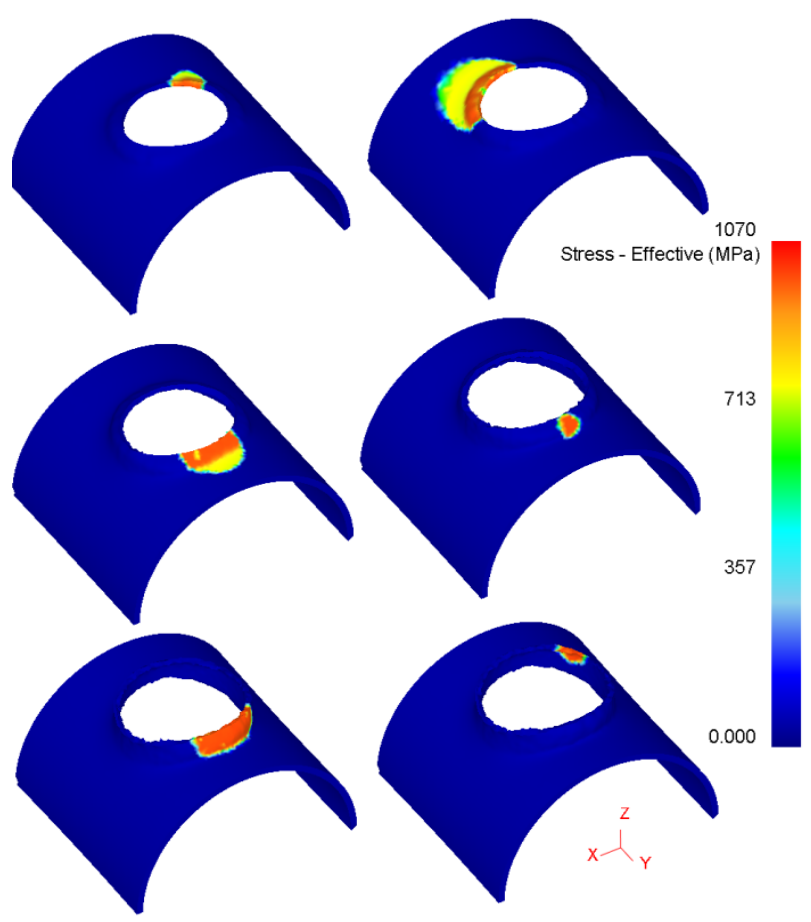

Fig.3. Progression of shape and distribution of effective stress during Ø50 collar forming into $\varnothing 150 \mathrm{~mm}$ pipe 


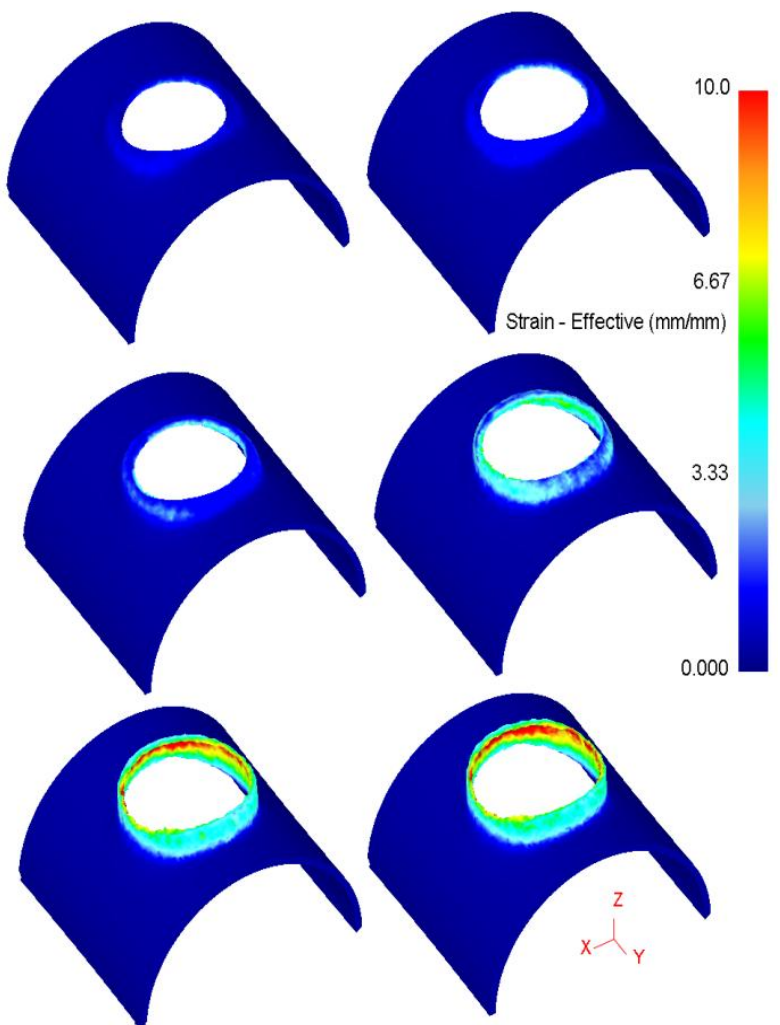

Fig.4. Progression of shape and distribution of effective strain during Ø50 collar forming into Ø150 mm pipe

As it is shown in Fig. 3 the distribution of effective stress is really local and maximum values of this parameter exceed 1000MPa. In zones of contact between tools and workpiece material is moved towards the direction of axial movement of tools. Presented in Fig. 4 effective strain distributions show real range of material deformation during flanging process. Values of this parameter are slightly different according to number of moving tools but the distributions are depended on different feed rates applications Bigger values of feed rate $\mathrm{f}=1$ and $2 \mathrm{~mm}$ per rotation provide to obtaining effective strain values reaching $12 \div 14$. Normally this information is important considering fine grain material structures into plastically formed zones. However this observation must be verified in experimental tests in real flanging collars device [5].

\subsection{Wall thickness and damage criterion distributions}

The wall thickness distributions in longitudinal and cross sections of chosen case are shown in Fig. 5.

Presented results show very favourable distribution of wall thickness. In all analysed zones of collar forming there are no significant decreases of this parameter. Very similar situation is observed in real case of collar forming by two rotating bars in steel P355NH pipe which is shown in Fig. 6. This information is very important for the future scope of use of T-pipe joint in welding of high pressure pipe ducts with very elevated mechanical characteristics.

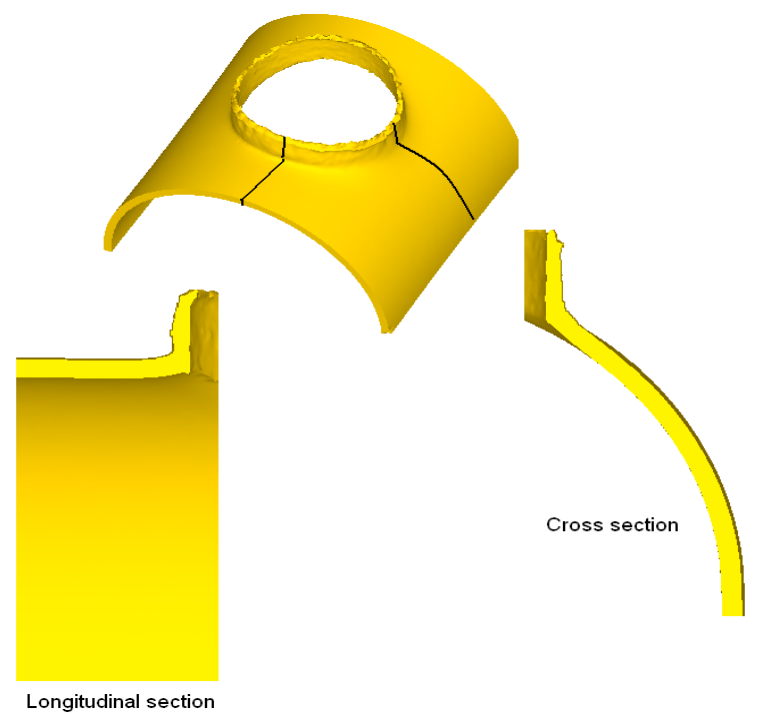

Fig.5. Wall thickness shapes - calculation results during Ø50 collar formed into Ø150 mm pipe

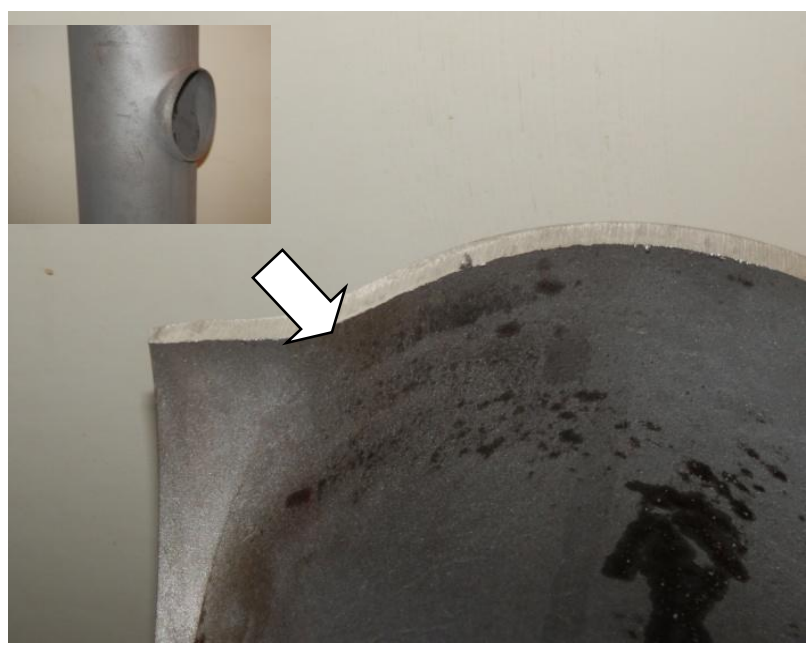

Fig.6. Wall thickness shape in cross section - real part view during Ø50 collar formed into Ø150 mm pipe

The differences between calculated and obtained in experiment distributions of collar wall thickness are not significant. The application of pipe with initial wall thickness $4.00 \mathrm{~mm}$ results in minimal calculated value $3.48 \mathrm{~mm}$ and measured in experiment $3.56 \mathrm{~mm}$. During the process observation it was stated that the friction conditions are very important for this values distribution. The application of old pipes with rusted inner surface in experiments results in bigger wall thinning during flange forming. The application of parts from construction steel with analogical dimensions and process conditions results in final collar wall thickness equal $3.16 \mathrm{~mm}$. This shows the importance of friction phenomena reduction during process realization. In the further research this value will be determined in experiments.

The distributions of damage criterion by Cockroft Latham were also analysed during calculations. One chosen distribution of this parameter at the final stage of forming is presented in Fig. 7. The obtained results confirmed that flanged material should not be damaged 
by internal defects especially by cracking. In the case of lower feed rate values as $\mathrm{f}=0.5 \mathrm{~mm} /$ rot. $\mathrm{C}$-L criterion integral reaches 0.8 comparing with $0.3 \div 0.4$ in case of bigger analysed feed rates.

\subsection{Load parameters}

Load parameters as axial forming force and torque were also analysed during simulations. Values of these parameters for the extreme conditions of forming are presented in Fig. 8 and Fig. 9.

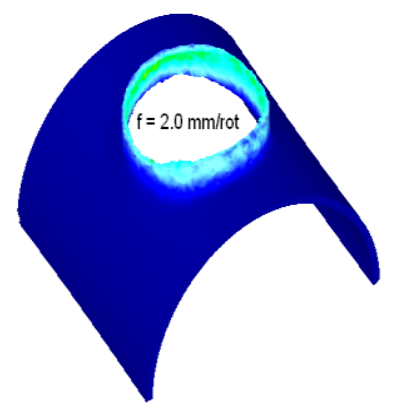

Damage
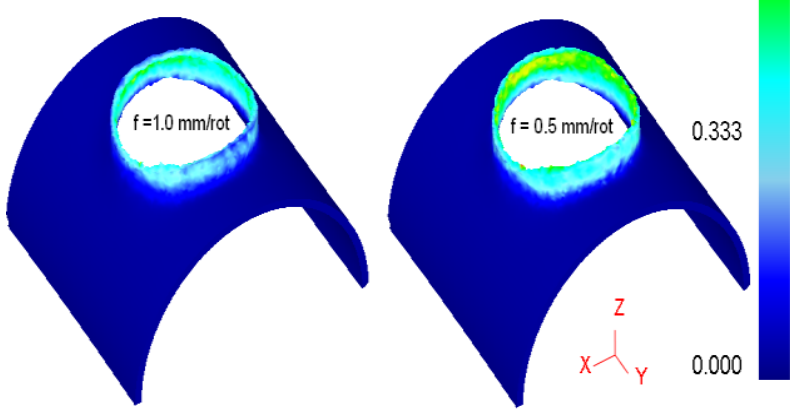

Fig.7.Cockroft-Latham damage criterion values for different feed rates - calculation results during Ø50 collar forming into $\varnothing 150 \mathrm{~mm}$ pipe

\section{Force, kN}

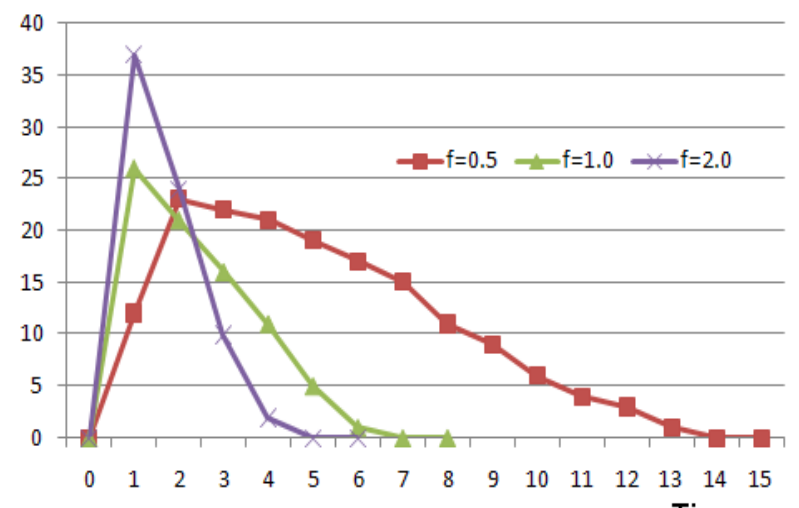

Time,s

Fig.8.Forming forces during collar forming - distributions with different feed rates

Numerical simulations results give information about values of forming forces and torques which is necessary in constrains for the future device designing. The knowledge of basic technological parameters and its influencing each other is very important for the process designing.

\section{Torque, $\mathrm{Nm}$}

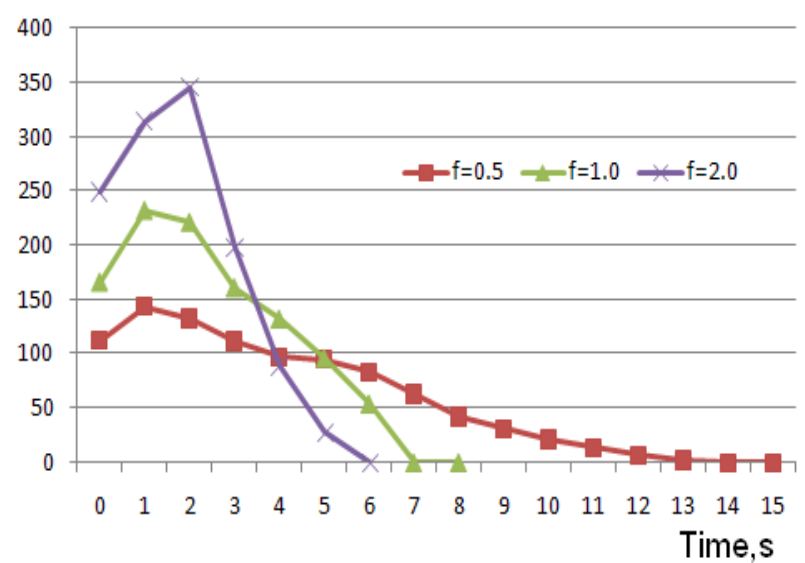

Fig.9. Torques during collar forming - distributions with different feed rates

\section{Conclusions}

Worked out numerical calculations of T-pipe joints forming show practical possibility of this process application. Regarding the existing solutions for this type of parts manufacturing it should be interesting to develop special device for this activity dedicated to the hydraulic press or for handy operated devices for smaller pipe diameters.

Some results concerning especially feed rate $f$ and inclination angle $\alpha$ and initial elliptic hole dimensions will be very useful for this process recognizing. The observed significant torque values reduction depending on applied feed rates will be very useful for handy operated portable device. In this case, the crucial limitation is pipe fixation during collar forming in existing installation. The device body stiffness is also very important because it will decide about whole dimensions accuracy and tools positions stability.

All results must be verified in experiments, It is especially important in the case of microstructure, hardness distribution and eventually internal defects very difficult to forecast by numerical calculations.

Another very important factor are friction conditions determining parts wall thickness distributions important for certification procedures of these T-joints.

Finally, all these issues will decide on the scope of the proposed solution applicability and safety rules for users.

\section{References}

1. https://t-drill.com/technologies/tee-forming/

2. Montanari L, Cristino VA, Silva MB, Martins PAF Int J Adv Manuf Technol 69(5) (2013)

3. Thipprakmas S, Phanitwong W Int $\mathbf{J}$ Adv Manuf Technol 61(5) (2012)

4. Cao TT, Lu B, Ou H, Long H, Chen J Int J Mach Tools Manuf 110(2016)

5. Yang C, Wen T, Liu LT, Wang H J Mat Process Technol 214(2014) 